\title{
Quantifying the Quality of Service of Streaming Media in Differentiated Services Networks
}

\author{
D.K. Agrawal ${ }^{1}$, T. Kleiberg ${ }^{1}$, S. Papp ${ }^{1}$, R.E. Kooij ${ }^{1,2}$, P. Van Mieghem ${ }^{1}$ \\ ${ }^{1}$ Faculty of Electrical Engineering Mathematics and Computer Science \\ Delft University of Technology, P.O. Box 5031, 2600 GA Delft, The Netherlands \\ \{D.K.Agrawal, T.J.Kleiberg, P.VanMieghem\} @tudelft.nl \\ ${ }^{2}$ TNO Information and Communication Technology \\ Brassersplein 2, P.O. Box 5050, 2600 GB Delft, The Netherlands \\ Robert.Kooij@tno.nl
}

\begin{abstract}
Quality of Service (QoS) support in the current internet is indispensable because of QoS-sensitive real-time applications such as Voice-over-IP, IP-TV, video conferencing, online gaming etc. Since the introduction of the Differentiated Services (DiffServ) architecture there has been considerable work reported in literature on its performance evaluation. However, none of them have addressed the basic issue of quantification of QoS for supporting streaming media. The main contribution in this paper is the quantification of the QoS of streaming media in terms of Mean Opinion Score (MOS) values. A test bed has been implemented using off-the-shelf components. The experimental results and MOS values are used to show that in a DiffServ Assured Forwarding network architecture, with class based weighted fair queue scheduling discipline, the QoS of streaming media is not compromised when the load exceeds the reserved capacity, even in case of congestion.
\end{abstract}

\section{INTRODUCTION}

Internet's traditional best-effort service model cannot provide guarantees required by streaming video, Voice-over-IP (VoIP) and quality sensitive business applications. Therefore it is necessary to offer service differentiation based on the requirements of users and applications. The Differentiated Services (DiffServ) architecture, see [26], has recently become the preferred method to address QoS issues in IP networks. This packet marking based approach to IP-QoS is attractive due to its simplicity and scalability. An endto-end differentiated service is obtained by concatenation of per-domain services and Service Level Agreements (SLA) between adjoining domains along the path that the traffic crosses in going from source to destination. Per domain services are realized by traffic conditioning at the edge and simple differentiated forwarding mechanisms at the core of the network. Two of the more popular proposed forwarding mechanisms are Expedited Forwarding (EF) and Assured Forwarding (AF) per hop behavior (PHB). The basic concept of AF-based services is simple mark and drop mechanisms to assure QoS. The AF approach will provide better than besteffort service by controlling the drop preference of packets at the time of congestion. In the presented work we have implemented the DiffServ AF architecture to ensure the QoS of streaming media in presence of other interfering traffic, including VoIP and FTP traffic. In recent years, experimental work on DiffServ architectures, is mainly reported in terms of network parameters, such as e.g. throughput, delay, jitter.

A number of simulation and experimental studies have carried out performance evaluation of DiffServ architectures. Bless et al. [1] illustrate some of the problems which will arise when IP Multicast is used in DiffServ networks without taking special precautions into account for providing it. These problems mainly lead to situations in which other service users are affected adversely. The discussion on the related problems and presentation of the solution is illustrated and confirmed by measurements performed with a Linux implementation of DiffServ and an adapted Linux Multicast Router. Hong Yu et al. [2] have used MPEG-2 video streams for evaluating QoS support in DiffServ. Their results give a very promising picture which describes that with proper resource management and traffic engineering, DiffServ can provide satisfactory QoS to applications with critical delay and delay variation, packet loss and throughput requirements, such as MPEG-2 video. Rogers et al. [3] demonstrated that UDP streams can be successfully mixed with TCP traffic in an Assured Forwarding context by policing the individual streams at the edge of a DiffServ area.

Qyou Zhou et al. [4] focus on analyzing the performance of real-time applications over a DiffServ-capable network, especially when real-time traffic aggregation and link congestion occur in a core router. Based on extensive testing results the authors propose a novel scheduling scheme, which can solve the problem generated by EF traffic aggregation and link congestion. Zhao Chen et al. [5] present simulation results assessing the performance of DiffServ-capable networks supporting real-time multimedia traffic using weighted fair queueing (WFQ). They evaluate the performance of voice, H.263 video, Distributed Interactive Virtual Environments (DIVE) applications and web like traffic in a DiffServ-capable network. Their analysis demonstrates that these multimedia applications can be well supported by DiffServ-capable networks even under bursty web traffic, given that the subscribed bandwidth of the applications is not less than its source generating data rate. Ali et al. [6] evaluated a DiffServ architecture with different queuing disciplines. As a result of their simulation they show that WFQ scheduling with well 
chosen weights performs better than FIFO and PQ. WenShyang Hwang et al. [7] propose a QoS-aware Residential Gateway (QRG) with real-time traffic monitoring, DiffServQoS and CBQ bandwidth management. Their results show that in both implementations, QRG successfully performs DiffServ-QoS and CBQ bandwidth management functions so that good QoS and video performance are maintained during network congestion. Dekeris et al. [8] also use a DiffServ architecture with WFQ. It is established, that at times of congestion, WFQ is not capable to implement DiffServ. Therefore combined WFQ and Low Latency Queue (LLQ) scheduling disciplines are used to ensure QoS for highest priority bursty video conferencing, voice and data services at the same time. The end-to-end video quality is determined not only by the network QoS but also by the encoded video quality. Hong Zhao et al. [9] considered video quality from these two aspects and proposed to transport spatial scalable encoded videos with different dropping priorities over DiffServ to provide QoS guarantees.

In this paper we will quantify the QoS of streaming video and VoIP as it will be experienced by users. The users' quality of experience will be expressed as a Mean Opinion Score (MOS), see [22]. The rest of the paper is organized as follows. Section 2. briefly describes the basic concept of class based weighted fair queueing and gives some background about MOS values. Test bed implementation is described in Section 3. and the experimental results are discussed in Section 4.. The conclusions of the paper are given in Section 5. .

\section{BACKGROUND}

Class-based weighted fair queuing (CBWFQ), see [11], extends the standard WFQ functionality to provide support for user defined traffic classes. For CBWFQ, traffic classes are defined based on match criteria including protocols, access control lists and input interfaces. Packets satisfying the match criteria for a class constitute the traffic for that class. A queue is reserved for each class, and traffic belonging to a class is directed to the queue for that class. Once a class has been defined according to its match criteria, the bandwidth, weight, and maximum packet limit is assigned. The bandwidth assigned to a class is the guaranteed bandwidth delivered to the class during congestion. To characterize a class, the queue limit for that class is defined. After a queue has reached its configured queue limit, enqueuing of additional packets to the class causes packet drop, depending on class policy configuration. Tail drop is default for CBWFQ classes. All unclassified flows are given best-effort treatment. If no default class is configured, then by default the traffic that does not match any of the configured classes is flow classified and given best-effort treatment. Once a packet is classified, all of the standard mechanisms that can be used to differentiate the service level among the classes apply. For CBWFQ, packets that arrive at the output interface are classified according to the match criteria filters, and then each one is assigned the appropriate weight. The weight for a packet belonging to a specific class is derived from the bandwidth assigned to the
Table 1 - Mean Opinion Score

\begin{tabular}{|c|l|}
\hline MOS & Quality Degradation \\
\hline 5 & Excellent, Not noticeable \\
4 & Good, Noticeable but not annoying \\
3 & Fair, Slightly annoying \\
2 & Poor, Annoying \\
1 & Bad, Very annoying \\
\hline
\end{tabular}

class when it is configured; in this sense the weight for a class is user-configurable. After the weight for a packet is assigned, the packet is queued in the appropriate class queue. CBWFQ uses the weights assigned to the queued packets to ensure that the class queue is served fairly. Taking into account available bandwidth on the interface, up to 64 classes can be configured and the distribution among them can be controlled, which is not the case with flow-based WFQ. Flow-based WFQ applies weights to traffic to classify it into separate classes and determine how much bandwidth each class is allowed relative to other classes. For flow-based WFQ, these weights, and the traffic classification, are dependent on and limited to the seven IP Precedence levels.

In multimedia (audio, voice, or video), especially when codecs are used to compress the bandwidth requirement, the Mean Opinion Score (MOS) provides a numerical indication of the perceived quality of received media after compression and/or transmission. The MOS is expressed as a single number in the range 1 to 5 , where 1 is lowest perceived quality, and 5 is the highest perceived quality. The meaning of the MOS scale, see Table 1, is specified in ITU-T Recommendation P.800 [22].

We will determine MOS values for streaming video by applying VQM, see [18]. VQM is a full reference method, i.e. VQM computes the quality of a transmitted video clip by comparing it to its corresponding original. The actual quality model within VQM that we used is the General Model, see also ITU-R BT.1683 [23] and ITU-T J.144 [24]. It should be noted that VQM only assesses the visual quality of the video. Thus, the audio quality and the synchronization between audio and video are not covered by VQM. The highest quality according to VQM is a MOS value of 5. To obtain MOS values for VoIP we will use the so-called E-model, defined in ITU-T Recommendation G.107 [25]. The E-model maps network, application and terminal parameters to MOS. In this paper we have used the freeware E-model implementation provided at [19]. Note that the highest quality according to the E-model is a MOS value of 4.41.

\section{NETWORK ARCHITECTURE}

The experimental test bed is implemented in the network laboratory of the Department of Telecommunications of Delft University of Technology, The Netherlands. The network topology is shown in Figure 1. It is a widely used de-facto single bottleneck topology for evaluating DiffServ architectures. We have used four Cisco [12] 2621XM routers using IOS (tm) C2600 Software (C2600-IS-M), Version 12.2(15)T11, 


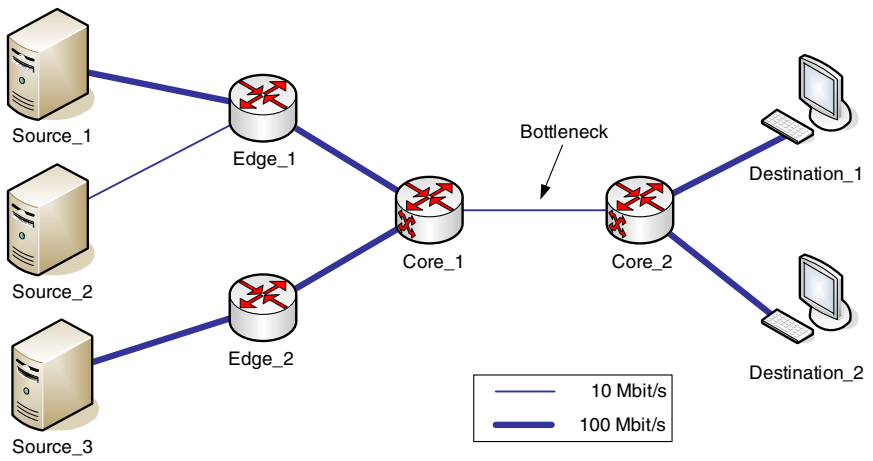

Figure 1 - Network topology of the test bed.

RELEASE SOFTWARE (fc2). There are five personal computers (PC); Intel Pentium-4 3.0 GHz machines with standard Ethernet interfaces and $512 \mathrm{MB}$ of RAM. All the PCs have Ubuntu Linux operating system version 6.06. Two out of five PCs are receivers (Destination_1 and Destination_2) and the other three PCs are sources (Source_1, Source_2 and Source_3) generating different types of traffic. The routers that are directly connected to the traffic sources are the edge routers. The role of the edge router is to mark packets according to source and destination IP address, source port and destination port numbers or protocol ID. The packets are individually marked by setting the DSCP field in the IP header. The other two routers are the core routers. The core routers classify the packets based on the DSCP value and forward the packets according to their configuration.

There are three types of traffic: DVD quality movie streams, VoIP and TCP/FTP. All these three type of applications have different DSCP values and bandwidth allocation. Source_1 generates video streams and TCP/FTP, Source_2 and Source_3 generate the VoIP traffic. The capacities of the links between the source PCs and the edge routers and the links between the edge routers and the core router are 100Mbps. The bottleneck link capacity between the two core routers is 10Mbps. The link between Source_2 and the Edge_1 router is 10Mbps because the Cisco 2621XM router has only two 100Mbps ports. But this does not affect the experiment because the traffic on this link is less than 10Mbps. Packet marking at the edge routers are done like [11], [12], [13], i.e. all the video streams are marked as DSCP AF21, all the VoIP flows are marked as DSCP AF11 and because the TCP/FTP traffic was not marked, it is treated as best-effort (DSCP BE).

At the core router we used CBWFQ [13] which allows different bandwidth allocation for different services. The sum of all bandwidth allocation on an interface cannot exceed 75 percent of the total available bandwidth. The remaining 25 percent is used for signaling, exchanging routing information and best-effort traffic. By default, traffic that does not match any of the configured classes is given best-effort treatment. For each measurement a warm-up period of 60 seconds is considered to allow the traffic to reach a steady-state. The measurement period was 300 seconds during which the data and video streams were recorded for 120 seconds. Then offline post-processing of data is performed. Cricket [15] and Nfdump/NfSen [16], [17] are used for traffic monitoring. The network monitoring tools collect and display information about the network traffic.

\section{EXPERIMENTS, RESULTS AND DISCUSSION}

We have performed a number of experiments to evaluate the CBWFQ DiffServ Assured Forwarding architecture. There are three network conditions: under provisioned network, exact provisioned network and over provisioned network. In case of under provisioning the bottleneck link capacity is less than the total traffic offered to the network, while in case of exact provisioning the bottleneck link capacity equals the total traffic load. In case of over provisioning the bottleneck link capacity exceeds the total amount of traffic fed into the link. We will discuss the various experiments performed for the above stated three network conditions later in this section. We have used constant bit rate video streams at $1.75 \mathrm{Mbps}$ per stream using the VLC media player [14], VoIP traffic at $80 \mathrm{Kbps}$ per call using the D-ITG traffic generator [20] and TCP/FTP traffic for all the experiments. The bottleneck link capacity is $10 \mathrm{Mbps}$ in all cases. The routers are work conservative, therefore unutilized link capacity, i.e. capacity that is not used in a certain class, is not wasted but used by other traffic classes.

\subsection{Experiment 1}

The first experiment is performed with one video stream, a number of VoIP flows and best effort TCP/FTP traffic. The bandwidth reservation for the video stream is varied between $15 \%, 17 \%, 18 \%$ and $20 \%$ while the corresponding reservation for VoIP flows is $60 \%, 58 \%, 57 \%$, and $55 \%$, respectively. The VoIP reservation had to be reduced, for increasing reservation for the video stream, because not more than $75 \%$ of the total link capacity can be reserved. Table 2 shows the results of the first experiment. Columns 1 and 2 show the link reservation in percentages for video and VoIP traffic, respectively. Column 3 indicates the number of VoIP flows used. The total traffic load offered to router 1 is shown in column 4 . Columns 5 and 6 list the MOS values for video and VoIP, respectively. MOS is calculated for video streams of one minute, captured at the destination. The MOS values for VoIP are based upon the E-model, where we have used measured network quality and the assumption that the used voice codec is G.711, with packet loss concealment implemented.

\subsection{Experiment 2}

In the second experiment two video clips are streamed, together with a number of VoIP flows and best effort TCP/FTP traffic. The bandwidth reservation for the video streams is varied between $25 \%, 30 \%, 35 \%$ and $40 \%$ while the corresponding reservation for VoIP is $50 \%, 45 \%, 40 \%$, and $35 \%$, respectively. Table 3 shows the results of Experiment 2 . The columns 1, 2, 3, 4 and 6 denote the same quantities as in the previous experiment. Column 5 shows the MOS values for both video streams (S1 and S2) used in the experiment. 
Table 2 - MOS values with single video stream

\begin{tabular}{|c|c|c|c|c|c|}
\hline $\begin{array}{c}\text { Reserv. } \\
\text { Video }\end{array}$ & $\begin{array}{c}\text { Reserv. } \\
\text { VoIP }\end{array}$ & $\begin{array}{l}\text { Traffic } \\
\text { Detail }\end{array}$ & \begin{tabular}{|l} 
Traffic \\
(Mbps)
\end{tabular} & $\begin{array}{l}\text { MOS } \\
\text { Video }\end{array}$ & $\begin{array}{l}\text { MOS } \\
\text { VoIP }\end{array}$ \\
\hline \multirow{3}{*}{$15 \%$} & \multirow{3}{*}{$60 \%$} & $\begin{array}{c}70 \text { VoIP } \\
2 \text { FTP }\end{array}$ & $\begin{array}{c}7.35 \\
+ \text { FTP }\end{array}$ & 5 & 4.41 \\
\hline & & $\begin{array}{c}80 \text { VoIP } \\
2 \text { FTP }\end{array}$ & $\begin{array}{c}8.15 \\
+\mathrm{FTP}\end{array}$ & 5 & 4.41 \\
\hline & & $\begin{array}{c}90 \text { VoIP } \\
2 \text { FTP }\end{array}$ & $\begin{array}{c}8.95 \\
+ \text { FTP }\end{array}$ & 1.68 & 3.93 \\
\hline \multirow{3}{*}{$17 \%$} & \multirow{3}{*}{$58 \%$} & $\begin{array}{c}70 \text { VoIP } \\
2 \text { FTP }\end{array}$ & $\begin{array}{c}7.35 \\
+\mathrm{FTP}\end{array}$ & 5 & 4.41 \\
\hline & & $\begin{array}{c}80 \text { VoIP } \\
2 \text { FTP }\end{array}$ & $\begin{array}{c}8.15 \\
+\mathrm{FTP}\end{array}$ & 5 & 4.41 \\
\hline & & $\begin{array}{c}90 \text { VoIP } \\
2 \text { FTP }\end{array}$ & $\begin{array}{r}8.95 \\
+ \text { FTP }\end{array}$ & 4.92 & 3.76 \\
\hline \multirow{3}{*}{$18 \%$} & \multirow{3}{*}{$57 \%$} & $\begin{array}{c}70 \text { VoIP } \\
2 \text { FTP }\end{array}$ & $\begin{array}{c}7.35 \\
+\mathrm{FTP}\end{array}$ & 5 & 4.41 \\
\hline & & $\begin{array}{c}80 \text { VoIP } \\
2 \text { FTP }\end{array}$ & $\begin{array}{c}8.15 \\
+\mathrm{FTP}\end{array}$ & 5 & 4.41 \\
\hline & & $\begin{array}{c}90 \text { VoIP } \\
2 \text { FTP }\end{array}$ & $\begin{array}{c}8.95 \\
+\mathrm{FTP}\end{array}$ & 5 & 3.75 \\
\hline \multirow{3}{*}{$20 \%$} & \multirow{3}{*}{$55 \%$} & $\begin{array}{c}70 \text { VoIP } \\
2 \text { FTP }\end{array}$ & $\begin{array}{c}7.35 \\
+ \text { FTP }\end{array}$ & 5 & 4.41 \\
\hline & & $\begin{array}{c}80 \text { VoIP } \\
2 \text { FTP }\end{array}$ & $\begin{array}{c}8.15 \\
+ \text { FTP }\end{array}$ & 5 & 4.41 \\
\hline & & $\begin{array}{c}90 \text { VoIP } \\
2 \text { FTP }\end{array}$ & $\begin{array}{c}8.95 \\
+\mathrm{FTP}\end{array}$ & 5 & 3.75 \\
\hline
\end{tabular}

\subsection{Experiment 3}

In Experiment 3 three video clips are streamed, together with a number of VoIP flows and best effort TCP/FTP traffic. The bandwidth reservation for the video streams is varied between $45 \%, 50 \%, 55 \%$ and $60 \%$ and the corresponding reservation for VoIP flows is $30 \%, 25 \%, 20 \%$, and $15 \%$, respectively. Table 4 shows the results of Experiment 3 . Columns 1, 2, 3, 4 and 6 are the same as in the previous experiment. Column 5 shows the MOS values for all three streams (S1, S2 and S3) used in the experiment.

From the measurements we observed that the best-effort TCP/FTP traffic suffered most in case of congestion, and that the throughput reached zero. The voice and video traffic must compete for the remaining $25 \%$ unreserved capacity in case of congestion. From Tables 2, 3 and 4 it is clear that the video traffic is very sensitive to loss. E.g. in Table 2 at $17 \%$ reservation and 90 VoIP flows, the video traffic shows degradation while only 50kbps extra capacity is required on top of the reserved capacity. Hence, the VoIP traffic appears more robust against loss.

\section{CONCLUSIONS}

We have implemented a real, DiffServ-aware network, where three different media must compete for resources, namely streaming video, VoIP and TCP/FTP. The sensitivity
Table 3 - MOS values with two video streams

\begin{tabular}{|c|c|c|c|c|c|c|}
\hline \multirow{2}{*}{$\begin{array}{c}\text { Reserv. } \\
\text { Video }\end{array}$} & \multirow{2}{*}{$\begin{array}{c}\text { Reserv. } \\
\text { VoIP }\end{array}$} & \multirow{2}{*}{$\begin{array}{l}\text { Traffic } \\
\text { Detail }\end{array}$} & \multirow{2}{*}{$\begin{array}{l}\text { Traffic } \\
\text { (Mbps) }\end{array}$} & \multicolumn{2}{|c|}{ MOS Video } & \multirow{2}{*}{$\begin{array}{l}\text { MOS } \\
\text { VoIP }\end{array}$} \\
\hline & & & & S1 & S2 & \\
\hline \multirow{3}{*}{$25 \%$} & \multirow{3}{*}{$50 \%$} & $\begin{array}{c}50 \text { VoIP } \\
2 \text { FTP }\end{array}$ & $\begin{array}{c}7.5 \\
+ \text { FTP }\end{array}$ & 5 & 5 & 4.41 \\
\hline & & $\begin{array}{c}60 \text { VoIP } \\
2 \text { FTP }\end{array}$ & $\begin{array}{c}8.3 \\
+ \text { FTP }\end{array}$ & 5 & 5 & 4.41 \\
\hline & & $\begin{array}{c}70 \text { VoIP } \\
2 \text { FTP }\end{array}$ & $\begin{array}{c}9.1 \\
+\mathrm{FTP}\end{array}$ & 1 & 1 & 4.41 \\
\hline \multirow{4}{*}{$30 \%$} & \multirow{4}{*}{$45 \%$} & $\begin{array}{c}50 \text { VoIP } \\
2 \text { FTP }\end{array}$ & $\begin{array}{c}7.5 \\
+\mathrm{FTP}\end{array}$ & 5 & 5 & 4.41 \\
\hline & & $\begin{array}{c}60 \text { VoIP } \\
2 \text { FTP }\end{array}$ & $\begin{array}{c}8.3 \\
+\mathrm{FTP}\end{array}$ & 5 & 5 & 4.41 \\
\hline & & $\begin{array}{c}70 \text { VoIP } \\
2 \text { FTP }\end{array}$ & $\begin{array}{c}9.1 \\
+\mathrm{FTP}\end{array}$ & 2.15 & 2.05 & 4.41 \\
\hline & & $\begin{array}{c}80 \text { VoIP } \\
2 \text { FTP }\end{array}$ & $\begin{array}{c}9.9 \\
+\mathrm{FTP}\end{array}$ & 2.15 & 2.10 & 4.41 \\
\hline \multirow{4}{*}{$35 \%$} & \multirow{4}{*}{$40 \%$} & \begin{tabular}{|c|}
50 VoIP \\
2 FTP
\end{tabular} & $\begin{array}{c}7.5 \\
+\mathrm{FTP}\end{array}$ & 5 & 5 & 4.41 \\
\hline & & $\begin{array}{c}60 \text { VoIP } \\
2 \text { FTP }\end{array}$ & $\begin{array}{c}8.3 \\
+\mathrm{FTP}\end{array}$ & 5 & 5 & 4.33 \\
\hline & & $\begin{array}{c}70 \text { VoIP } \\
2 \text { FTP }\end{array}$ & $\begin{array}{c}9.1 \\
+\mathrm{FTP}\end{array}$ & 5 & 5 & 3.42 \\
\hline & & $\begin{array}{c}80 \text { VoIP } \\
2 \text { FTP }\end{array}$ & $\begin{array}{c}9.9 \\
+\mathrm{FTP}\end{array}$ & 5 & 5 & 2.56 \\
\hline \multirow{4}{*}{$40 \%$} & \multirow{4}{*}{$35 \%$} & \begin{tabular}{|c|}
50 VoIP \\
2 FTP
\end{tabular} & $\begin{array}{c}7.5 \\
+\mathrm{FTP}\end{array}$ & 5 & 5 & 4.41 \\
\hline & & \begin{tabular}{|c|}
60 VoIP \\
2 FTP
\end{tabular} & $\begin{array}{c}8.3 \\
+ \text { FTP }\end{array}$ & 5 & 5 & 4.33 \\
\hline & & $\begin{array}{c}70 \text { VoIP } \\
2 \text { FTP }\end{array}$ & $\begin{array}{c}9.1 \\
+\mathrm{FTP}\end{array}$ & 5 & 5 & 3.41 \\
\hline & & $\begin{array}{c}80 \text { VoIP } \\
2 \text { FTP }\end{array}$ & $\begin{array}{c}9.9 \\
+\mathrm{FTP}\end{array}$ & 5 & 5 & 2.56 \\
\hline
\end{tabular}

of the media to network congestion is studied and the effect of loss on the perceived QoS is evaluated. Assessment of the QoS was performed with the use of Mean Opinion Scores, which provide an indication for the experienced QoS by the end user. Our experiments have shown that if the aggregated data rate within a traffic class does not exceed the allocated bandwidth in that class, that the QoS can be guaranteed, even in case of congestion of the link. Moreover, we argue that CBWFQ can be used to guarantee QoS in commercially available routers. In case the reserved bandwidth for both video and VoIP is under provisioned, then the quality degradation for video is more severe. The reason is that video is more sensitive for packet loss than VoIP. A future direction in the line of this work is to assess the quality of experience for TCP/FTP or web traffic. In addition, the user experienced quality for Online Gaming could be taken into account as well. 
Table 4 - MOS values with three video streams

\begin{tabular}{|c|c|c|c|c|c|c|c|}
\hline \multirow{2}{*}{$\begin{array}{c}\text { Reserv. } \\
\text { Video }\end{array}$} & \multirow{2}{*}{$\begin{array}{c}\text { Reserv. } \\
\text { VoIP }\end{array}$} & \multirow{2}{*}{$\begin{array}{l}\text { Traffic } \\
\text { Detail }\end{array}$} & \multirow{2}{*}{\begin{tabular}{|l} 
Traffic \\
(Mbps)
\end{tabular}} & \multicolumn{3}{|c|}{ MOS Video } & \multirow{2}{*}{$\begin{array}{l}\text { MOS } \\
\text { VoIP }\end{array}$} \\
\hline & & & & S1 & S2 & S3 & \\
\hline \multirow{5}{*}{$45 \%$} & \multirow{5}{*}{$30 \%$} & $\begin{array}{c}30 \text { VoIP } \\
2 \text { FTP }\end{array}$ & $\begin{array}{c}7.65 \\
+ \text { FTP }\end{array}$ & 5 & 5 & 5 & 4.41 \\
\hline & & $\begin{array}{c}40 \text { VoIP } \\
2 \text { FTP }\end{array}$ & $\begin{array}{r}8.45 \\
+ \text { FTP }\end{array}$ & 4.94 & 4.93 & 4.58 & 4.41 \\
\hline & & $\begin{array}{c}50 \text { VoIP } \\
2 \text { FTP }\end{array}$ & $\begin{array}{l}9.25 \\
+\mathrm{FTP}\end{array}$ & 2.24 & 2.59 & 3.21 & 3.37 \\
\hline & & $\begin{array}{c}60 \text { VoIP } \\
2 \text { FTP }\end{array}$ & $\begin{array}{l}10.05 \\
+ \text { FTP }\end{array}$ & 2 & 2.74 & 2.81 & 2.34 \\
\hline & & $\begin{array}{c}70 \text { VoIP } \\
2 \text { FTP }\end{array}$ & $\begin{array}{l}10.85 \\
+ \text { FTP }\end{array}$ & 2 & 1.9 & 2.56 & 1.93 \\
\hline \multirow{5}{*}{$50 \%$} & \multirow{5}{*}{$25 \%$} & $\begin{array}{c}30 \text { VoIP } \\
2 \text { FTP }\end{array}$ & $\begin{array}{r}7.65 \\
+\mathrm{FTP}\end{array}$ & 5 & 5 & 5 & 4.41 \\
\hline & & $\begin{array}{c}40 \text { VoIP } \\
2 \text { FTP }\end{array}$ & $\begin{array}{c}8.45 \\
+ \text { FTP }\end{array}$ & 5 & 5 & 5 & 4.27 \\
\hline & & $\begin{array}{c}50 \text { VoIP } \\
2 \text { FTP }\end{array}$ & $\begin{array}{l}9.25 \\
+\mathrm{FTP}\end{array}$ & 5 & 5 & 5 & 2.92 \\
\hline & & \begin{tabular}{|c|}
60 VoIP \\
2 FTP
\end{tabular} & $\begin{array}{l}10.05 \\
+\mathrm{FTP}\end{array}$ & 5 & 5 & 5 & 2.15 \\
\hline & & \begin{tabular}{|c|}
70 VoIP \\
2 FTP
\end{tabular} & $\begin{array}{l}10.85 \\
+\mathrm{FTP}\end{array}$ & 5 & 5 & 5 & 1.83 \\
\hline \multirow{5}{*}{$55 \%$} & \multirow{5}{*}{$20 \%$} & $\begin{array}{c}30 \text { VoIP } \\
2 \text { FTP }\end{array}$ & $\begin{array}{c}7.65 \\
+ \text { FTP }\end{array}$ & 5 & 5 & 5 & 4.41 \\
\hline & & $\begin{array}{c}40 \text { VoIP } \\
2 \text { FTP }\end{array}$ & $\begin{array}{r}8.45 \\
+ \text { FTP }\end{array}$ & 5 & 5 & 5 & 4.15 \\
\hline & & $\begin{array}{c}50 \text { VoIP } \\
2 \text { FTP }\end{array}$ & $\begin{array}{r}9.25 \\
+ \text { FTP }\end{array}$ & 5 & 5 & 5 & 2.91 \\
\hline & & $\begin{array}{c}60 \text { VoIP } \\
2 \text { FTP }\end{array}$ & $\begin{array}{l}10.05 \\
+ \text { FTP }\end{array}$ & 5 & 5 & 5 & 2.14 \\
\hline & & \begin{tabular}{|c|}
70 VoIP \\
2 FTP
\end{tabular} & $\begin{array}{l}10.85 \\
+\mathrm{FTP}\end{array}$ & 5 & 5 & 5 & 1.82 \\
\hline \multirow{5}{*}{$60 \%$} & \multirow{5}{*}{$15 \%$} & $\begin{array}{c}30 \text { VoIP } \\
2 \text { FTP }\end{array}$ & $\begin{array}{c}7.65 \\
+ \text { FTP }\end{array}$ & 5 & 5 & 5 & 4.39 \\
\hline & & $\begin{array}{c}40 \text { VoIP } \\
2 \text { FTP }\end{array}$ & $\begin{array}{c}8.45 \\
+\mathrm{FTP}\end{array}$ & 5 & 5 & 5 & 4.11 \\
\hline & & $\begin{array}{c}50 \text { VoIP } \\
2 \text { FTP }\end{array}$ & $\begin{array}{l}9.25 \\
+ \text { FTP }\end{array}$ & 5 & 5 & 5 & 2.89 \\
\hline & & \begin{tabular}{|c|}
60 VoIP \\
2 FTP
\end{tabular} & $\begin{array}{l}10.05 \\
+\mathrm{FTP}\end{array}$ & 5 & 5 & 5 & 2.14 \\
\hline & & $\begin{array}{c}70 \text { VoIP } \\
2 \text { FTP }\end{array}$ & $\begin{array}{l}10.85 \\
+\mathrm{FTP}\end{array}$ & 5 & 5 & 5 & 1.82 \\
\hline
\end{tabular}

\section{ACKNOWLEDGMENT}

This work has been partially supported by the Dutch funding agency for university research (STW) under project "Network Dynamics and QoS", DTC.6421.

\section{REFERENCES}

[1] L. Bless and R. Wehrle: "Group communication in differentiated services networks", IEEE/ACM International Symposium on Cluster Computing and the Grid, 2001, p.p. 618-625

[2] H. Yu, D. Makrakis and L.O. Barbosa: "Experimental evaluation of MPEG-2 video over differentiated services IP networks", IEEE Pacific Rim Conference on Communications, Computers and signal Processing, 2001. Vol. 2, August 2628, 2001, p.p. 469-472.

[3] G. Rogers, M. Minhazuddin and R. Liu: "Mixing UDP and TCP in a DiffServ assured forwarding PHB - a programmable networks scenario", IEEE International Conference on Networks, Oct. 10-12, 2001 p.p. 529-534.

[4] Q. Zhou, H. Yu, D, Makrakis and O. Kabranov, L. OrozcoBarbosa: "Performance evaluation for real-time traffic in Differentiated Services capable IP network", IEEE Canadian Conference on Electrical and Computer Engineering, Vol. 3, May 12-15, 2002, p.p. 1522-1526.

[5] Z. Chen, T. Z. Yang and D. Makrakis: "Performance analysis of multimedia applications in Differentiated Services networks" IEEE Canadian Conference on Electrical and Computer Engineering, Vol. 3, May 12-15, 2002, p.p. 1594-1599.

[6] R.B. Ali, S. Pierre and Y. Lemieux: "DiffServ QoS performance evaluation of multimedia telephony", Canadian Conference on Electrical and Computer Engineering, Vol. 4, May 2-5, 2004, p.p. 2115-2118.

[7] W-S. Hwang and P-C. Tseng: "A QoS-aware residential gateway with bandwidth management", IEEE Transactions on Consumer Electronics, Vol. 51, Issue 3, Aug. 2005, p.p. 840848.

[8] B. Dekeris, T. Adomkus and A. Budnikas: "Analysis of QOS assurance using weighted fair queuing scheduling discipline with low latency queue", International Conference on Information Technology Interfaces, 2006, p.p. 507-512.

[9] H. Zhao, N. Ansari and Y. Q. Shi: "Layered MPEG video transmission over IP DiffServ", International Conference on Information Technology: Coding and Computing, 2005, Vol. 1, April 4-6, 2005, p.p. 63-67.

[10] Z. Avramova, D. De Vleeschauwer, S. Wittevrongel and H. Bruneel: "Multiplexing Gain of Capped VBR Video", Workshop on QoS and Traffic Control, Sep. 7-9, 2005.

[11] http://www.cisco.com/univercd/cc/td/doc/product/software/ ios120/120newft/120t/120t5/cbwfq.htm.

[12] http://www.cisco.com/en/US/products/hw/routers/ps259/ products_tech_note09186a0080094e92.shtml.

[13] http://www.cisco.com/univercd/cc/td/doc/product/software/ ios122/122cgcr/fqos_c/fqcprt2/qcfconmg.htm\#wp1001203.

[14] http://www.videolan.org/vlc/.

[15] http://cricket.sourceforge.net/.

[16] http://nfdump.sourceforge.net/.

[17] http://nfsen.sourceforge.net/.

[18] http://www.its.bldrdoc.gov/n3/video/VQM_software.php.

[19] http://davidwall.com/MOSCalc.htm.

[20] http://www.grid.unina.it/software/ITG/citings.php.

[21] ITU-T, "Transmission impairments due to speech processing", (ITU-T G.113), 2001

[22] ITU-T, "Methods for Subjective Determination of Transmission Quality", (ITU-T P.800), 1996.

[23] ITU-R, "Objective perceptual video quality measurement techniques for standard definition digital broadcast television in the presence of a full reference", (ITU-R BT.1683) Geneva, Switzerland, 2004.

[24] ITU-T, "Objective perceptual video quality measurement techniques for digital cable television in the presence of a full reference", (ITU-T J.144), 2004.

[25] ITU-T, "The E-model, a computational model for use in transmission planning", (ITU-T G.107), 2003.

[26] D. Black, S. Blake, M. Carlson, E. Davies, Z. Wang, and W. Weiss, "An Architecture for Differentiated Services", (IETF RFC 2475), Dec. 1998. 